\title{
Hamilton cycles in random graphs with a fixed degree sequence
}

\author{
Colin Cooper* Alan Frieze ${ }^{\dagger} \quad$ Michael Krivelevich ${ }^{\ddagger}$
}

March 22, 2010

\begin{abstract}
Let $\mathbf{d}=d_{1} \leq d_{2} \leq \cdots \leq d_{n}$ be a non-decreasing sequence of $n$ positive integers, whose sum is even. Let $\mathcal{G}_{n, \mathbf{d}}$ denote the set of graphs with vertex set $[n]=\{1,2, \ldots, n\}$ in which the degree of vertex $i$ is $d_{i}$. Let $G_{n, \mathbf{d}}$ be chosen uniformly at random from $\mathcal{G}_{n, \mathbf{d}}$. It will be apparent from Section 4.3 that the sequences we are considering will all be graphic. We give a condition on $\mathbf{d}$ under which we can show that whp $\mathcal{G}_{n, \mathbf{d}}$ is Hamiltonian. This condition is satisfied by graphs with exponential tails as well those with power law tails.
\end{abstract}

\section{Introduction}

Let $\mathbf{d}=d_{1} \leq d_{2} \leq \cdots \leq d_{n}$ be a fixed non-decreasing sequence of $n$ positive integers, whose sum is even. Let $\mathcal{G}_{n, \mathbf{d}}$ denote the set of graphs with vertex set $V=[n]=\{1,2, \ldots, n\}$ in which the degree of vertex $i$ is $d_{i}$. Let $G_{n, \mathbf{d}}$ be chosen uniformly at random from $\mathcal{G}_{n, \mathbf{d}}$. It will be apparant from Section 4.3 that the sequences we are considering will all be graphic. When $d_{i}=r$ for $i \in[n]$ then this models a random $r$-regular graph $G_{n, r}$ and there is a large literature on this subject. We refer the reader to the survey by Wormald [15] for an excellent summary. By now we know much about the structure of random regular graphs.

For general $\mathbf{d}$, less is known. In many, but not all, cases we can estimate $\left|\mathcal{G}_{n, \mathbf{d}}\right|$; see Bender and Canfield [2], McKay and Wormald [9, 10]. We have the configuration model to study

\footnotetext{
*Department of Computer Science, King's College, University of London, London WC2R 2LS, UK

$\dagger$ 'Department of Mathematical Sciences, Carnegie Mellon University, Pittsburgh PA15213, U.S.A. Supported in part by NSF grant CCF-0502793.

${ }^{\ddagger}$ Department of Mathematics, Raymond and Beverly Sackler Faculty of Exact Sciences, Tel Aviv University, Tel Aviv 69978, Israel. E-mail: krivelev@post.tau.ac.il. Research supported in part by USA-Israel BSF Grant 2002-133 and by grants 64/01 and 526/05 from the Israel Science Foundation.
} 
them, Bollobás [3]. We know something of their connectivity properties, Molloy and Reed $[12,13]$ and Cooper [5]. (See also Cooper and Frieze [6] for the connectivity properties of random digraphs with a fixed degree sequence). They have been used in the context of massive graph models of telephone networks and the WWW, Aiello, Chung and Lu [1].

In a previous paper [7] we studied the chromatic number of $G_{n, \mathbf{d}}$. Let

$$
D_{k}=d_{n}+d_{n-1}+\cdots+d_{n-k+1}
$$

be the sum of the $k$ largest degrees.

Let $d$ denote the average degree and let

$$
M_{1}=D_{n}=d n \text { and } M_{2}=\sum_{i=1}^{n} d_{i}\left(d_{i}-1\right) \leq \Delta M_{1} \text { where } \Delta=d_{n} .
$$

We proved the following:

Theorem 1 [7]

1. Suppose that there exist constants $1 / 2<\alpha<1, \epsilon, K>0$ and $\omega=\omega(n) \rightarrow \infty$ such that

(a)

$$
D_{t} \leq K d n(t / n)^{\alpha}
$$

$$
\text { for } t \leq \epsilon n
$$

(b)

$$
\Delta^{5} \leq M_{2} / \omega
$$

Then there exists $b_{1}$ dependent only on $\alpha, \epsilon, K$ such that $\mathbf{w h}^{1}$

$$
\chi\left(G_{n, \mathbf{d}}\right) \leq b_{1} \frac{d}{\ln d} .
$$

2. Suppose only that $\Delta^{4} \leq M_{1} / \omega$ (a weaker condition than $1(b)$ ), then there exists $b_{2}$ such that whp

$$
\chi\left(G_{n, \mathbf{d}}\right) \geq b_{2} \frac{d}{\ln d}
$$

Condition 1(b) is required for the use the results of [11]. We will make the same assumption when we deal with Hamiltonicity. It may be possible to prove our results under the less stringent conditions of [10], but there are difficulties, as will be pointed to later.

\footnotetext{
${ }^{1}$ A sequence of events $\mathcal{E}_{n}, n \geq 0$ is said to occur with high probability $(\mathbf{w h p})$ if $\lim _{n \rightarrow \infty} \operatorname{Pr}\left(\mathcal{E}_{n}\right)=1$.
} 
It is natural to ask whether there many types of degree sequences that satisfy the conditions of the first part of the theorem. It is easy to see that regular graphs are included. In [7] we showed that degree sequences satisfying (1) are important. We considered those with power law and exponential tails and showed that they satisfied the conditions of Theorem 1 :

Power Law Tails: For integer $\ell \geq 1$ we let $\nu_{\ell}$ denote the number of vertices of degree $\ell$. Our assumption is that there are some constants $A>0$ and $\zeta>3$ such that for $\ell \geq(A / \epsilon)^{1 /(\zeta-1)}$

$$
\nu_{\ell} \leq \begin{cases}0 & \ell \leq 1 \\ \left\lfloor A d \ell^{-\zeta} n\right\rfloor & 2 \leq \ell \leq n^{1 / 5} / \ln n . \\ 0 & \ell>n^{1 / 5} / \ln n\end{cases}
$$

Here we have $\alpha=\frac{\zeta-2}{\zeta-1}>1 / 2$.

Exponential Tails: For some constants $A>0$ and $0<\epsilon \ll \zeta<1$ we have for $\ell \geq$ $\left\lfloor\log _{1 / \zeta}(A d / \epsilon)\right\rfloor$

$$
\nu_{\ell} \leq A d \zeta^{\ell} n .
$$

Note that whp the degree sequence of $G_{n, p}, p=c / n, c$ constant, satisfies this condition.

In this paper we study the Hamiltonicity of $G_{n, \mathbf{d}}$. We prove the following:

Theorem 2 Suppose that there exist constants $1 / 2<\alpha<1, \epsilon, K>0$ and $\omega=\omega(n) \rightarrow \infty$ such that (1) and (2) hold. Suppose also that

A1 $d_{1} \geq K_{1} d^{1-1 / 4 \alpha}$ for sufficiently large $K_{1}$.

A2 $d \leq n^{\gamma}$, where $\gamma$ is constant and $\gamma<\frac{2 \alpha-1}{2 \alpha+1}$.

A3 $d$ is sufficiently large.

Then $G_{n, \mathbf{d}}$ is Hamiltonian whp.

\section{Configurations}

We will work initially in the configuration model and then show how our result can be justified in the uniform model, i.e. $G_{n, \mathbf{d}}$. Let $W=[n d]$ be our set of points and let $W_{i}=\left[d_{1}+\cdots+d_{i-1}+1, d_{1}+\cdots+d_{i}\right], i \in[n]$, partition $W$. The function $\phi: W \rightarrow[n]$ is defined by $w \in W_{\phi(w)}$. Given a pairing $F$ (i.e. a partition of $W$ into $m=d n / 2$ pairs) we obtain a (multi-)graph $G_{F}$ with vertex set $[n]$ and an edge $(\phi(u), \phi(v))$ for each $\{u, v\} \in F$. Choosing a pairing $F$ uniformly at random from among all possible pairings of the points of $W$ produces a random (multi-)graph $G_{F}$. 
This model is valuable because of the following easily proven fact: Suppose $G \in \mathcal{G}_{n, \mathbf{d}}$. Then

$$
\operatorname{Pr}\left(G_{F}=G \mid G_{F} \text { is simple }\right)=\frac{1}{\left|\mathcal{G}_{n, \mathbf{d}}\right|} .
$$

It follows that if $G$ is chosen randomly from $\mathcal{G}_{n, \mathbf{d}}$, then for any graph property $\mathcal{P}$

$$
\operatorname{Pr}(G \in \mathcal{P}) \leq \frac{\operatorname{Pr}\left(G_{F} \in \mathcal{P}\right)}{\operatorname{Pr}\left(G_{F} \text { is simple }\right)}
$$

In the next section it will be useful to consider that $F$ is constructed by taking a random permutation $\psi$ of $W$ and then taking $F=\left\{e_{j}=\{\psi(2 j-1), \psi(2 j)\}: j=1,2, \ldots, m\right\}$. We can then for example use the following version of the Azuma-Hoeffding concentration inequality. Let random variable $Z=Z(\pi)$ be such that if $\pi^{\prime}$ is obtained from $\pi$ by a single inversion then $\left|Z(\pi)-Z\left(\pi^{\prime}\right)\right| \leq \rho$. Then

$$
\operatorname{Pr}(|Z-\mathbf{E}(Z)| \geq t) \leq 2 e^{-t^{2} / m \rho^{2}} .
$$

For a proof, see for example Lemma 11 of [8].

\section{$3 \quad$ Non-Hamiltonian Degree Sequences}

We observe next that there are simple degree sequences for which $G_{n, \mathbf{d}}$ is non-Hamiltonian whp.

Let $\nu=\lceil 2 n / 3\rceil$ and let $d_{1}=d_{2}=\cdots=d_{\nu}=h$ and $d_{\nu+1}=d_{\nu+2}=\cdots=d_{n}=D$ for some $h, D$ independent of $n$. Let $d=(\nu h+(n-\nu) D) / n$ be the average degree. In the configuration model we have $\operatorname{Pr}\left(G_{F}\right.$ is simple $)=\Omega(1)$.

Let $F$ be chosen randomly. Suppose that $D \gg h$. Let $V_{1}=[\nu]$ and $V_{2}=[n] \backslash V_{1}$. Next let

$$
Z_{1}=\mid\left\{j \in V_{1}: j \text { only has neighbours in } V_{2}\right\} \mid \text {. }
$$

Now

$$
\mathbf{E}\left(Z_{1}\right)=\nu \frac{((n-\nu) D)((n-\nu) D-1) \cdots((n-\nu) D-h+1)}{(d n-1)(d n-3) \cdots(d n-2 h+1)} \sim \frac{2}{3}\left(\frac{D}{D+2 h}\right)^{h} n .
$$

An application of (4) can be used to show that $Z_{1} \sim \frac{2}{3}\left(\frac{D}{D+2 h}\right)^{h} n$ whp. Now choose $D, h$ such that $\left(\frac{D}{D+2 h}\right)^{h}>\frac{1}{2}$. Note that $D=L h^{2}$ for large $L$ will suffice.

Then whp $G_{F}$ contains an independent subset inside $V_{1}$ with more than $\left|V_{2}\right|$ vertices. Clearly $G_{F}$ is non-Hamiltonian in this case.

It is important to see how the above degree sequence violates the conditions of Theorem 2 . We can choose $K, \epsilon, \alpha$ such that (1) and (2) hold, but we will find that $d_{1}=h$ is too small to satisfy Condition A1 of the theorem when $d$ is large. 


\section{Proof of Theorem 2}

We randomly and independently colour the elements of $W$ Red, Blue and Yellow, each with probability $1 / 3$. We use this colouring of $W$ to induce a colouring of the edges of $G_{F}$. The Red edges will form an expander. The Blue edges will be used to ensure connectivity and the Yellow edges will be used to complete a Hamilton cycle. Let $W_{R}$ be the set of Red elements and let $\widehat{F}_{R}=\left\{e \in F: e \cap W_{R} \neq \emptyset\right\}$ be the set of edges that are immediately coloured Red i.e. the edges for which at least one of its configuration points is Red. Let $\widehat{G}_{R}=\left(V, \widehat{F}_{R}\right)$ be the subgraph of $G_{F}$ induced by $\widehat{F}_{R}$. For $S \subseteq F$ and $i \in[n]$ let $d(i, S)=\left|\left\{e \in S: e \cap W_{i} \neq \emptyset\right\}\right|$. Then let $V_{0}=\left\{i: d\left(i, \widehat{F}_{R}\right) \leq d_{1} / 2\right\}$ and let $F_{R}=\widehat{F}_{R} \cup\left\{e \in F: e \cap V_{0} \neq \emptyset\right\}$ be the final set of Red $G_{F}$ edges. Let $G_{R}=\left(V, F_{R}\right)$ be the subgraph of $G_{F}$ induced by $F_{R}$. Note that $G_{R}$ has minimum degree at least $d_{1} / 2$.

Fix a configuration $F$. We first observe that with $\operatorname{Bin}(n, p)$ being the binomially distributed random variable with parameters $n$ and $p$,

$$
\operatorname{Pr}\left(i \in V_{0} \mid F\right) \leq \operatorname{Pr}\left(\operatorname{Bin}\left(d_{i}, \frac{4}{9}+\frac{d_{i}}{M_{1}-d_{i}}\right) \geq \frac{d_{i}}{2}\right)
$$

where the $\frac{d_{i}}{M_{1}-d_{i}}$ term accounts for the degree loss due to loops. Thus by Chernoff bounds,

$$
\mathbf{E}\left(\left|V_{0}\right| \mid F\right) \leq \sum_{i=1}^{n} e^{-d_{i} / 200}
$$

Now changing the colour of an element of $W$ changes $\left|V_{0}\right|$ by at most 2 and so it follows from Azuma's inequality that for any $t>0$,

$$
\operatorname{Pr}\left(\left|V_{0}\right| \geq \sum_{i=1}^{n} e^{-d_{i} / 200}+t \mid F\right) \leq e^{-t^{2} / 2 d n}
$$

So we see that whp,

$$
\left|V_{0}\right| \leq n_{0}=n e^{-d_{1} / 200}+(d n \log n)^{1 / 2}
$$

\subsection{Expansion of $G_{R}$}

For $X \subseteq F$ and $S \subseteq[n]$ let $N_{X}(S)$ be the set of vertices which are not in $S$, but have a $G_{X}$ neighbour in $S$ where $G_{X}=([n], X)$. We abbreviate $N_{F_{R}}(S)$ to $N_{R}(S)$. Our aim is to prove

\section{Lemma 1}

$$
\text { Whp }\left|N_{R}(S)\right| \geq 2|S| \text { for all } S \subseteq[n],|S| \leq s_{1}
$$


where

$$
\frac{s_{1}}{n}=\min \left\{\left(\frac{d_{1}}{32 d K^{2} e^{1+20 / d_{1}}}\right)^{2 \alpha-1-20 / d_{1}},(2 K)^{-1 / \alpha}, \epsilon\right\} .
$$

At this point we remark that if $K_{1}$ is sufficiently large then Condition A1 of Theorem 2 implies

$$
s_{1}^{2} d_{1}^{2} \geq 2000 d n^{2} .
$$

This inequality is enough to verify (17) below.

Proof

Case 1: $|S| \leq s_{0}$ where

$$
s_{0}=\left(12 d K^{2} e^{1+20 / d_{1}}\right)^{-1 /\left(2 \alpha-1-20 / d_{1}\right)} n .
$$

Let $\mathcal{E}_{S}$ be the event that $S$ contains at least $d_{1}|S| / 20$ edges in $G_{F}$. Let $d_{S}=\sum_{i \in S} d_{i}$. Then

$$
\begin{aligned}
\operatorname{Pr}\left(\bigcup_{|S| \leq s_{0}} \mathcal{E}_{S}\right) & \leq \sum_{s=3}^{s_{0}} \sum_{|S|=s}\left(\begin{array}{c}
d_{S} \\
d_{1} s / 10
\end{array}\right) \frac{\left(d_{1} s / 10\right) !}{\left(d_{1} s / 20\right) ! 2^{d_{1} s / 20}}\left(\frac{1}{d n-d_{1} s / 10}\right)^{d_{1} s / 20} \\
& \leq \sum_{s=3}^{s_{0}} \sum_{|S|=s} \frac{d_{S}^{d_{1} s / 10}}{\left(d_{1} s / 20\right) ! 2^{d_{1} s / 20}}\left(\frac{1}{d n-d_{1} s / 10}\right)^{d_{1} s / 20} \\
& \leq \sum_{s=3}^{s_{0}} \sum_{|S|=s}\left(\frac{d_{S}^{2} e}{d_{1} s / 10} \times \frac{1}{d n-d_{1} s / 10}\right)^{d_{1} s / 20} \\
& \leq \sum_{s=3}^{s_{0}}\left(\begin{array}{c}
n \\
s
\end{array}\right)\left(\frac{12 K^{2} d^{2} n^{2}(s / n)^{2 \alpha} e}{d_{1} s d n}\right)^{d_{1} s / 20} \\
& \leq \sum_{s=3}^{s_{0}}\left(\left(\frac{s}{n}\right)^{2 \alpha-1-20 / d_{1}} \frac{12 d K^{2} e^{1+20 / d_{1}}}{d_{1}}\right)^{d_{1} s / 20} \\
& =o(1) .
\end{aligned}
$$

Assume that $\mathcal{E}_{X}$ does not occur for $|X| \leq s_{0} . G_{R}$ is a subgraph of $G_{F}$ and so we can assume the corresponding event does not happen in $G_{R}$. Now suppose that $|S| \leq s_{0} / 3$ and $|T|<2|S|$ where $T=N_{R}(S)$. Let $p, q$ be the number of Red $G_{F}$ edges contained in $S$ and from $S$ to $T$ respectively. Then $2 p+q$ is equal to the total Red degree of $S$. Thus,

$$
2 p+q \geq d_{1}|S| / 2 \text { and } p \leq d_{1}|S| / 20 \text {. }
$$

But this implies that $p+q \geq 9 d_{1}|S| / 20>d_{1}|S \cup T| / 20$, a contradiction.

Remark 1 Suppose that we delete a set of edges with no vertex being incident to more than 20 and we add a set of edges with no vertex being incident to more than 25 of these. 
Then (11) would become $2 p+q \geq\left(d_{1}-40\right)|S| / 2$ and $p \leq\left(d_{1}+500\right)|S| / 20$. But this implies that $p+q \geq\left(9 d_{1}-900\right)|S| / 20>\left(d_{1}+500\right)|S \cup T| / 20$, and we get the same contradiction. This remark will be used in translating our result from configurations to $G_{n, \mathbf{d}}$. We will use a switching argument that whp involves the deletion (and addition) of o(s $\left.s_{0}\right)$ edges.

Case 2: $s_{0} / 3 \leq|S| \leq s_{1}$.

Note first that $d_{1} \gg \log d$ by assumption and we can then see from Condition A2 of Theorem 2 that $\left|V_{0}\right| \leq n_{0}=o\left(s_{0}\right)$. We will assume therefore from now on that $s_{0} / 3 \geq 100\left|V_{0}\right|$.

Fix sets $S, T$ with $|T|=2|S|=2 s$ and suppose that $N_{R}(S) \subseteq T$. Now $\left|V_{0}\right| \leq|S| / 100$ and so if $\widehat{d}_{S}$ denotes the total degree of $S$ in $\widehat{G}_{R}$ then $\widehat{d}_{S} \geq 99 d_{1} s / 200$.

Let $p, q$ be as above, but defined with respect to $\widehat{G}_{R}$. At this point we remark that $d_{S} \leq$ $d n / 2$, which follows from (1) and the second term in the expression (7) for $s_{1}$.

The probability $S$ contains $p$ edges of $\widehat{G}_{R}$ and there are $q$ edges of $\widehat{G}_{R}$ from $S$ to $T$ is at most

$$
\begin{aligned}
\left(\begin{array}{c}
d_{S} \\
2 p
\end{array}\right) \frac{(2 p) !}{p ! 2^{p}}\left(\frac{1}{d n-2 p+1}\right)^{p}\left(\frac{K d n(2 s / n)^{\alpha}}{d n-2 p}\right)^{q} & \leq \frac{2(d n)^{2 p+q}\left(K(s / n)^{\alpha}\right)^{2 p}}{p ! 2^{p}(d n-2 p)^{p+q}}\left(K(2 s / n)^{\alpha}\right)^{q} \\
& \leq\left(K(s / n)^{\alpha}\right)^{\widehat{d}_{S}} 2^{(1+\alpha) q}\left(\frac{d n e}{p}\right)^{p} \\
& \leq\left(K(s / n)^{\alpha}\right)^{\widehat{d}_{S}} 2^{(1+\alpha) q}\left(\frac{2 d n e}{\widehat{d}_{S}}\right)^{\widehat{d}_{S} / 2} \\
& \leq\left(\left(\frac{s}{n}\right)^{2 \alpha-1} \frac{32 K^{2} d e}{d_{1}}\right)^{\widehat{d}_{S} / 2} .
\end{aligned}
$$

Explanation for (12): We choose $2 p$ members of $W$ to make up the $\widehat{G}_{R}$ edges of $S$ in at most $\left(\begin{array}{l}d_{S} \\ 2 p\end{array}\right)$ ways. We choose a partition of these points into $p$ pairs in $\frac{(2 p) !}{p ! 2^{p}}$ ways. The quantity $1 /(d n-2 p+1)^{p}$ bounds the probability that these pairs exist in $\widehat{G}_{R}$. Then $\left(d_{T} /(d n-2 p)\right)^{q}$ bounds the probability that the remaining $q$ points are paired in $T$. We use (1) to bound $d_{T}$. We use $2 p+q \leq d_{S} \leq d n / 2$ to simplify the calculations.

So the probability that there exists such a pair $S, T$ is at most

$$
\begin{aligned}
& \sum_{p, q} \sum_{s=s_{0} / 3}^{s_{1}}\left(\begin{array}{l}
n \\
s
\end{array}\right)\left(\begin{array}{c}
n \\
2 s
\end{array}\right)\left(\left(\frac{s}{n}\right)^{2 \alpha-1} \frac{32 K^{2} d e}{d_{1}}\right)^{d_{1} s / 5} \\
& \leq d^{2} n^{2} \sum_{s=s_{0} / 3}^{s_{1}}\left(\left(\frac{s}{n}\right)^{2 \alpha-1-20 / d_{1}} \frac{32 K^{2} d e^{1+20 / d_{1}}}{d_{1}}\right)^{d_{1} s / 5} \\
& =o(1) .
\end{aligned}
$$


Remark 2 Replacing $\left(\begin{array}{c}n \\ 2 s\end{array}\right)$ by $\left(\begin{array}{c}n \\ 2.001 s\end{array}\right)$ in (13) allows us to claim that whp $\left|N_{R}(S)\right| \geq 2|S|$ in Case 2, even after deleting o $\left(s_{0}\right)$ edges. More precisely, the sum in (13) will evaluate to o(1). Thus we see that whp $\left|N_{R}(S)\right| \geq 2.001|S|$ for all $s_{0} / 3 \leq|S| \leq s_{1}$. Then after the deletion of $o\left(s_{0}\right)$ edges we will still have $\left|N_{R}(S)\right| \geq 2|S|$ for all $s_{0} / 3 \leq|S| \leq s_{1}$. This remark will be used in translating our result from configurations to $G_{n, \mathbf{d}}$. We will use a switching argument that whp involves the deletion (and addition) of o $\left(s_{0}\right)$ edges.

Going back to our construction of $G_{R}$, let $\widehat{F}_{B}$ denote the set of pairs $\{v, w\} \in F$ in which $v$ and $w$ are both coloured Blue and let $F_{B}=\left\{e=\{v, w\} \in \widehat{F}_{B}: \phi(v), \phi(w) \notin V_{0}\right\}$ where $\phi$ is defined in Section 2. Let $W_{B}=\bigcup_{e \in F_{B}} e$ and $V_{B}=\phi\left(W_{B}\right)$ and note that $F_{B}$ is a random pairing of $W_{B}$. Now let

$$
V_{1}=\left\{i \in[n]: d\left(i, F_{B}\right) \leq d_{1} / 10\right\} \subseteq V_{0} \cup N_{F}\left(V_{0}\right) \cup\left\{i \in[n]: d\left(i, \widehat{F}_{B}\right) \leq d_{1} / 10\right\} .
$$

Arguing as for (5) we have that whp

$$
\left|N_{F}\left(V_{0}\right)\right| \leq n d_{1} e^{-d_{1} / 200}+(d n \log n)^{1 / 2} \text { and }\left|\left\{i \in[n]: d\left(i, \widehat{F}_{B}\right) \leq d_{1} / 10\right\}\right| \leq n e^{-d_{1} / 1800},
$$

in which case we can assume that

$$
\left|V_{1}\right| \leq 2 n d_{1} e^{-d_{1} / 200}+2(d n \log n)^{1 / 2} \leq s_{1} .
$$

Definition 1 A graph $G=(V, E)$ is called a $(k, c)$-expander if $|N(U)| \geq c|U|$ for every subset $U \subseteq V(G)$ of cardinality $|U| \leq k$. (Here $N(S)$ is the set of vertices which are not in $S$, but have a neighbour in $S)$.

We have shown that whp $G_{R}$ is an $\left(s_{1}, 2\right)$-expander. Thus whp each component of $G_{R}$ has size at least $3 s_{1}$. We can now show that adding the extra edges $F_{B}$ will whp connect these components and thus show that $G_{R B}=G_{R}+F_{B}$ is connected whp. We will need to prove just a little more. Indeed, let $C_{1}, C_{2}, \ldots, C_{\rho}, \rho \leq n / 3 s_{1}$ be the components of $G_{R}$. We see from (14) that if $C_{i}^{\prime}=C_{i} \backslash V_{1}$ then $\left|C_{i}^{\prime}\right| \geq 2 s_{1}$ for $i=1,2, \ldots, \rho$. If $x \in W_{B}$ and $\phi(w) \in C_{i}^{\prime}$ then the probability it is paired with $y \in W_{B}, \phi(y) \in C_{j}$ is at least $\frac{s_{1} d_{1}-5 t}{5 d n}$ given that we have made $t$ pairings of $x \in W_{B}$. Thus by considering the first $s_{1} d_{1} / 10$ such pairings, we see that $\left|F_{B} \cap\left(C_{i} \times C_{j}\right)\right|$ dominates $\operatorname{Bin}\left(s_{1} d_{1} / 10, s_{1} d_{1} / 10 d n\right)$. Thus by a Chernoff bound,

$$
\begin{aligned}
& \operatorname{Pr}\left(G_{R B} \text { is not connected }\right) \leq \\
& \quad \operatorname{Pr}\left(\exists 1 \leq i<j \leq \rho:\left|F_{B} \cap\left(C_{i} \times C_{j}\right)\right| \leq \frac{s_{1}^{2} d_{1}^{2}}{200 d n}\right) \leq \frac{n^{2}}{9 s_{1}^{2}} \exp \left\{-\frac{s_{1}^{2} d_{1}^{2}}{800 d n}\right\}=o(1) .
\end{aligned}
$$




\subsection{Pósa's Lemma and its consequences}

Definition 2 Let $G=(V, E)$ be a non-Hamiltonian graph with a longest path of length

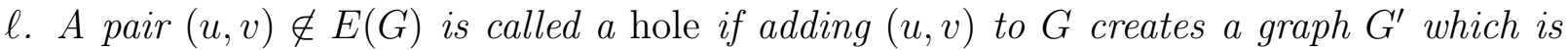
Hamiltonian or contains a path longer than $\ell$.

Lemma 2 Let $G$ be a non-Hamiltonian connected $(k, 2)$-expander. For every longest path of $G$ there is a set $A$ of size $k$ and sets $B_{a}, a \in A$, each of size $k$ such that $(a, b)$ is a hole for each $b \in B_{a}$.

Proof Let $P=\left(v_{0}, \ldots, v_{k}\right)$ be a longest path in graph $G$. A Pósa rotation of $P$ [14] with $v_{0}$ fixed gives another longest path $P^{\prime}=\left(v_{0}, \ldots v_{i} v_{k} \ldots v_{i+1}\right)$ created by adding edge $\left(v_{k}, v_{i}\right)$ and deleting edge $\left(v_{i}, v_{i+1}\right)$. Let $A=E N D_{G}\left(v_{0}, P\right)$ be the set of endpoints obtained by a sequence of Pósa rotations starting with $P$, keeping $v_{0}$ fixed and using an edge $\left(v_{k}, v_{i}\right)$ of $G$.

Each vertex $a \in A$ can then be used as the initial vertex of another set of longest paths whose endpoint set is $B_{a}=E N D_{G}\left(v_{j}, P\right)$, this time using $a$ as the fixed vertex, but again only adding edges from $G$.

The Pósa condition (see, e.g., [4], Ch.8.2)

$$
\left|N\left(E N D_{G}(v, P)\right)\right| \leq 2\left|E N D_{G}(v, P)\right|-1
$$

for $v \in E N D_{G}(P)$ together with the fact that $G$ is a $(k, 2)$-expander implies that $\left|E N D_{G}(v, P)\right|>k$. The connectivity of $G$ implies that closing a longest path to a cycle either creates a Hamilton cycle or creates a longer path. For every $v \in E N D_{G}(P)$ and for every $u \in E N D_{G}(v, P)$, a pair $(u, v)$ is a hole.

Going back to our construction of $\widehat{F}_{B}$, let $\widehat{F}_{Y}$ denote the set of pairs $\{v, w\} \in F$ in which $v$ and $w$ are coloured Yellow and let $F_{Y}=\left\{e=\{v, w\} \in \widehat{F}_{Y}: \phi(v), \phi(w) \notin V_{0}\right\}$. Let $W_{Y}=$ $\bigcup_{e \in F_{Y}} e$ and $V_{Y}=\phi\left(W_{Y}\right)$ and note that $F_{Y}$ is a random pairing of $W_{Y}$. Now let

$$
V_{2}=\left\{i \in[n]: d\left(i, F_{Y}\right) \leq d_{1} / 10\right\}
$$

Note that

$$
V_{2} \subseteq V_{0} \cup N_{F}\left(V_{0}\right) \cup\left\{i \in[n]: d\left(i, \widehat{F}_{Y}\right) \leq d_{1} / 10\right\} .
$$

Arguing as for (5) we have that whp

$$
\left|V_{2}\right| \leq 2 n d_{1} e^{-d_{1} / 200}+2 n^{3 / 4} \leq s_{1} / 2
$$

Suppose that $G_{R B}$ is not Hamiltonian. We start with a longest path $P$ of $G_{R B}$ and construct $A, B_{a}, a \in A$ as in Lemma 2 using only the edges of $G_{R B}$. Now choose $a \in A \backslash V_{2}$ and 
one by one expose the $F_{Y}$ pairings involving $W_{a}$. There are at least $d_{1} / 10$ (Yellow, Yellow) points to be paired and the probability of a pairing with a point in $W_{b}, b \in B_{a} \backslash V_{2}$ is at least $p_{t}=\frac{s_{1} d_{1} / 20-2 t-1}{d n-2 t-1}=\frac{s_{1} d_{1}-40 t-10}{20 d n-40 t-20}$, given $t$ previous attempts at such a pairing. If there is a pairing then we add the corresponding edge $e$ and either complete a Hamilton cycle or find a longer path $P^{\prime}$ in $G_{R B}+e$.

We can then repeat this process with $P^{\prime}$. We claim that whp we can continue this process until we have added enough Yellow edges to create a Hamilton cycle. To see this we couple the process with a sequence of Bernoulli trials where the probability of success is $p_{t}$. It is sufficient to show that whp there will be at least $n$ successes before we make $t_{1}=s_{1} d_{1} / 80$ trials. But $t \leq t_{1}$ implies $p_{t} \geq \frac{s_{1} d_{1}-40}{40 d n-s_{1} d-1-40}$ and so the expected number of successes in the first $t_{1}$ trials is at least

$$
\frac{t_{1}\left(s_{1} d_{1}-40\right)}{40 d n-s_{1} d_{1}-40} n \geq 2 n, \quad \text { see }(8) .
$$

We can therefore claim that whp there are at least $3 n / 2$ successes in the first $t_{1}$ trials and our claim follows.

This shows that $G_{F}$ is Hamiltonian whp. We now translate the result to $G_{n, \mathbf{d}}$.

\subsection{From configurations to graphs}

It is at this point that we appeal to some results from McKay and Wormald [11]. Where possible, we will use the terminology and notation of that paper. A loop of a pairing $F$ is a pair $\{u, v\}$ such that $\phi(u)=\phi(v)$. A double pair of $F$ is a pair of pairs $\left\{u_{1}, v_{1}\right\},\left\{u_{2}, v_{2}\right\} \in$ $F$ such that $\phi\left(u_{1}\right)=\phi\left(u_{2}\right)$ and $\phi\left(v_{1}\right)=\phi\left(v_{2}\right)$. A double loop of $F$ is a pair of pairs $\left\{u_{1}, v_{1}\right\},\left\{u_{2}, v_{2}\right\}$ such that $\phi\left(u_{1}\right)=\phi\left(v_{1}\right)=\phi\left(u_{2}\right)=\phi\left(v_{2}\right)$. A triple pair is a triple of pairs $\left\{u_{i}, v_{i}\right\}, i=1,2,3$ such that $\phi\left(u_{1}\right)=\phi\left(u_{2}\right)=\phi\left(u_{3}\right)$ and $\phi\left(v_{1}\right)=\phi\left(v_{2}\right)=\phi\left(v_{3}\right)$.

Condition (b) of Theorem 1 has played no part as yet. We do however need it to apply the results of [11]. So, in the lemmas that follow, we will assume that Condition (b) of Theorem 1 holds.

Lemma 3 (Lemma 2 of [11])

The probability that $F$ contains at least one triple pair is $O\left(\Delta^{2} M_{2}^{2} / M_{1}^{3}\right)=o(1)$ and the probability of at least one double loop is $O\left(\Delta^{2} M_{2} / M_{1}^{2}\right)=o(1)$.

Let now $l$ denote the number of loops and $r$ denote the number of double pairs in $F$.

Lemma 4 (Lemma 3 of [11])

If $\lambda(n) \rightarrow \infty$ then whp

$$
l \leq 2 \Delta+\lambda \text { and } r \leq \Delta^{2}+\lambda
$$




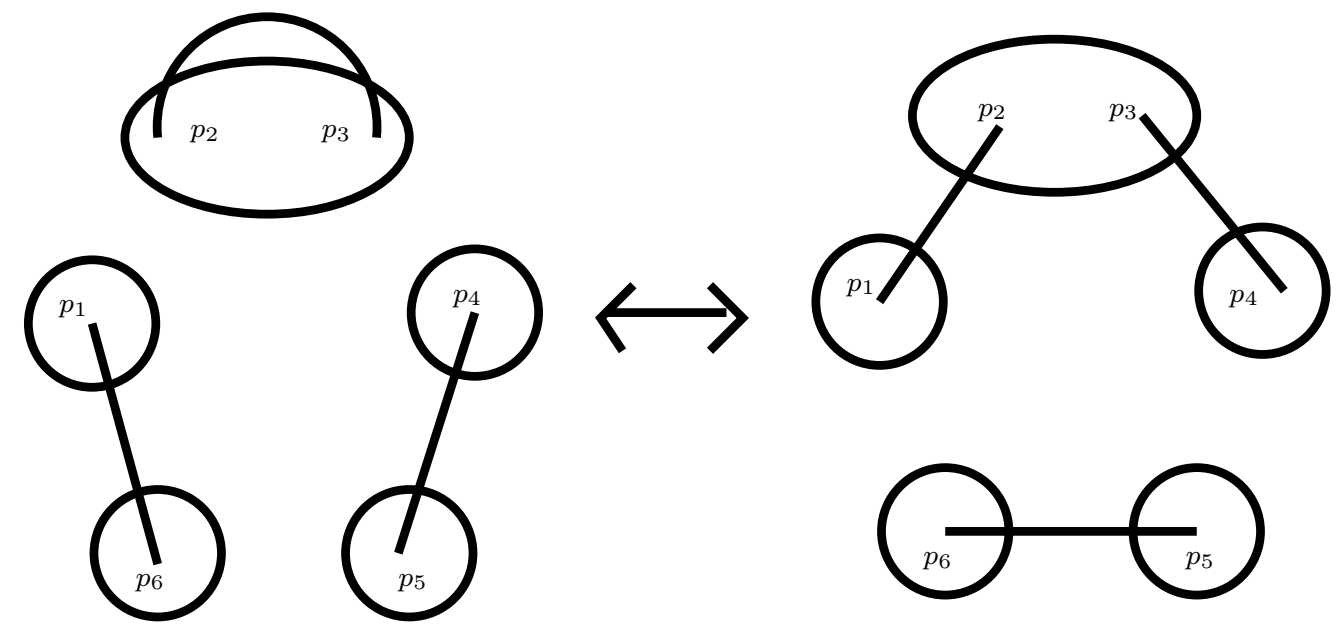

Figure 1:

We define the following two operations on a pairing: If $\phi(u)=i$ then we say that $u$ is in cell $i$.

\section{I l-switching.}

Take pairs $\left\{p_{1}, p_{6}\right\},\left\{p_{2}, p_{3}\right\},\left\{p_{4}, p_{5}\right\}$ where $\left\{p_{2}, p_{3}\right\}$ is a loop, and $p_{1}, \ldots, p_{6}$ are in five different cells. Replace these pairs by $\left\{p_{1}, p_{2}\right\},\left\{p_{3}, p_{4}\right\},\left\{p_{5}, p_{6}\right\}$. In this operation, none of the pairs created or destroyed is permitted to be part of a double pair. (See Figure 1).

II $r$-switching.

Take pairs $\left\{p_{1}, p_{5}\right\},\left\{p_{2}, p_{6}\right\},\left\{p_{3}, p_{7}\right\},\left\{p_{4}, p_{8}\right\}$ where $\phi\left(p_{2}\right)=\phi\left(p_{3}\right)$ and $\phi\left(p_{6}\right)=\phi\left(p_{7}\right)$, but the cells containing $p_{1}, p_{2}, p_{4}, p_{5}, p_{6}, p_{8}$ are all distinct. Replace these pairs by $\left\{p_{1}, p_{2}\right\},\left\{p_{3}, p_{4}\right\},\left\{p_{5}, p_{6}\right\},\left\{p_{7}, p_{8}\right\}$. In this operation, none of the pairs created or destroyed (other than the pairs $\left\{p_{2}, p_{6}\right\},\left\{p_{3}, p_{7}\right\}$ ) is permitted to be part of a multiple pair. (See Figure 2).

A forward $l$-switching is an $l$-switching as described, and a backward $l$-switching is the reverse operation. We use the same convention for $r$-switchings. Note that a forward $l$ switching always reduces the number of loops by one and does not create or destroy double pairs. Similarly, a forward $r$-switching reduces the number of double pairs by one and neither creates nor destroys loops. 

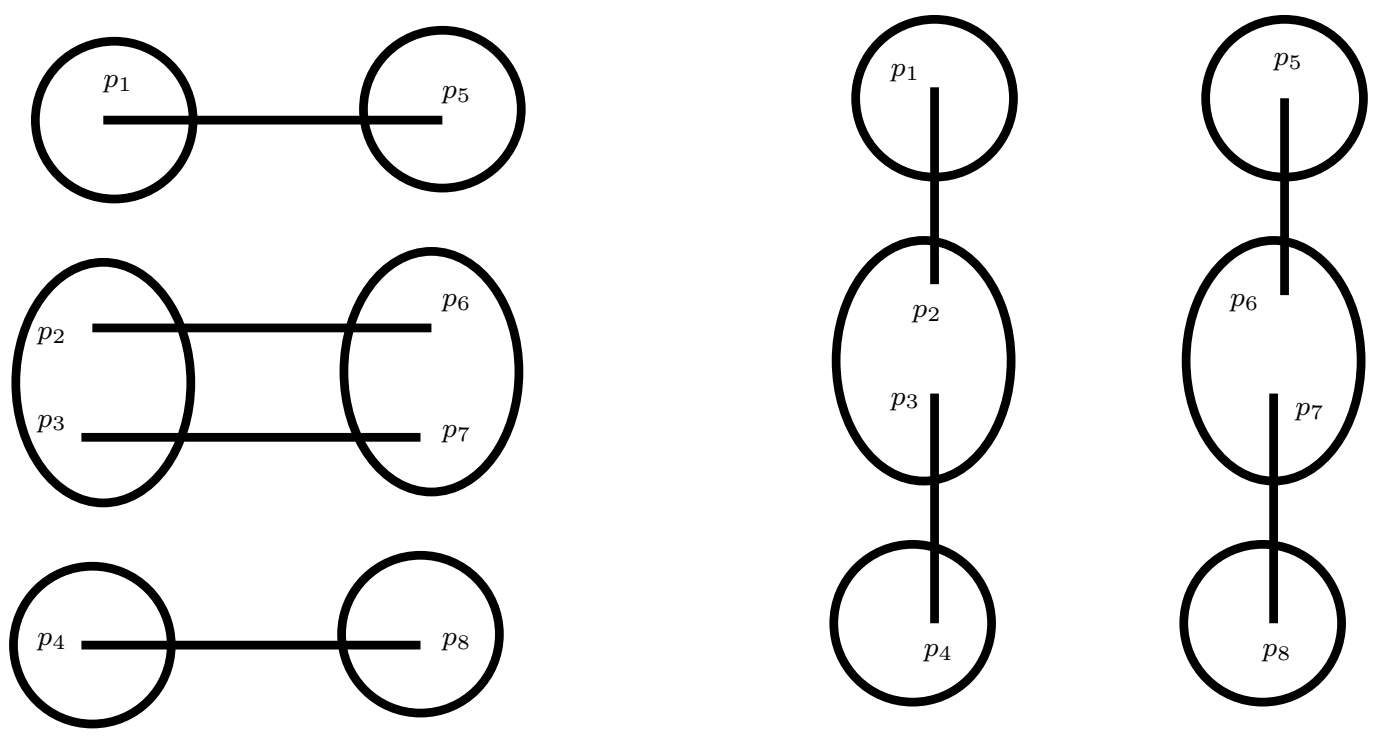

Figure 2:

Now let $\mathcal{C}_{l, r}$ denote the set of pairings $F$ with $l$ loops, $r$ double pairs and no triple pairs or double loops.

Lemma 5 (Lemma 4 of [11])

Denote an operation taking an element of $\mathcal{C}_{i, j}$ to an element $\mathcal{C}_{k, l}$ by $\mathcal{C}_{i, j} \rightarrow \mathcal{C}_{k, l}$. For each of the following operations, we bound the number, $m$, of ways of applying the operation to a fixed $F$.

(1) Forward l-switching $\mathcal{C}_{l, r} \rightarrow \mathcal{C}_{l-1, r}$ :

$$
2 l M_{1}^{2} \geq m \geq 2 l M_{1}^{2}\left(1-O\left(\frac{\Delta^{2}+l+r}{M_{1}}\right)\right) .
$$

(2) Backward l-switching $\mathcal{C}_{l-1, r} \rightarrow \mathcal{C}_{l, r}$ :

$$
M_{1} M_{2} \geq m \geq M_{1} M_{2}\left(1-\frac{\Delta(6(l+2 r)+\Delta l)}{M_{2}}-\frac{2 \Delta(\Delta+2)}{M_{1}}\right) .
$$

(3) Forward r-switching $\mathcal{C}_{0, r} \rightarrow \mathcal{C}_{0, r-1}$ :

$$
4 r M_{1}^{2} \geq m \geq 4 r M_{1}^{2}\left(1-O\left(\frac{\Delta^{2}+r}{M_{1}}\right)\right) .
$$

(4) Backward r-switching $\mathcal{C}_{0, r-1} \rightarrow \mathcal{C}_{0, r}$ :

$$
M_{2}^{2} \geq m \geq M_{2}^{2}\left(1-\frac{\Delta\left(16 r+9 \Delta+3+\Delta^{2}\right)}{M_{2}}\right) .
$$


Now consider the following algorithm for generating a member of $\mathcal{G}_{n, \mathrm{~d}}$ :

1. Generate a random pairing $F$.

2. If there is a double loop or a triple pair, output $\perp-$ construed as failure.

3. If the number of loops $l \geq 2 \Delta+\log n$ or the number of double pairs $r \geq \Delta^{2}+\log n$, output $\perp-$ construed as failure.

4. $F_{0} \leftarrow F$.

5. For $i=1$ to $l$ choose a random forward $l$-switching on $F_{i-1}$, creating $F_{i} \in \mathcal{C}_{l-i, r}$.

6. For $i=l+1$ to $l+r$ choose a random forward $r$-switching on $F_{i-1}$, creating $F_{i} \in$ $\mathcal{C}_{0, r-(i-l)}$.

7. Output $G^{*}=G_{F_{l+r}} \in \mathcal{G}_{n, \mathbf{d}}$.

For each $l, r$ satisfying (18), with $\lambda=\log n$, and $G \in \mathcal{G}_{n, \mathbf{d}}$, there are by Lemma $5(2),(4)$

$$
\left(M_{1} M_{2}\right)^{l} M_{2}^{2 r}\left(1+O\left(\frac{\Delta l(\Delta l+r)}{M_{2}}+\frac{\Delta^{2} l}{M_{1}}+\frac{\Delta r\left(\Delta^{2}+r\right)}{M_{2}}\right)\right)
$$

sequences of switchings which yield $G$. Each of these has probability

$$
\left(\left(2 M_{1}^{2}\right)^{l} l !\left(4 M_{1}^{2}\right)^{r} r !\right)^{-1}\left(1+O\left(\frac{l\left(\Delta^{2}+l+r\right)}{M_{1}}+\frac{r\left(\Delta^{2}+r\right)}{M_{1}}\right)\right)
$$

of being followed by the algorithm, given $l, r$.

Thus if Condition 1(b) holds, then whp the algorithm outputs a graph in $\mathcal{G}_{n, \mathbf{d}}$ and

$$
\operatorname{Pr}\left(G^{*}=G\right)=(1+o(1)) \sum_{l=0}^{2 \Delta+\log n} \sum_{r=0}^{\Delta^{2}+\log n} \frac{M_{1}^{l} M_{2}^{2 r+l}}{2^{l+2 r} M_{1}^{2(l+r)} l ! r !} \operatorname{Pr}(l \text { loops }, r \text { double pairs })
$$

and so for $G_{1}, G_{2} \in \mathcal{G}_{n, \mathbf{d}}$

$$
\operatorname{Pr}\left(G^{*}=G_{1}\right)=(1+o(1)) \operatorname{Pr}\left(G^{*}=G_{2}\right) .
$$

Given this, we only have to show that $\operatorname{whp} G^{*}$ is Hamiltonian.

Let $H_{1}$ be the graph consisting of those edges of $G_{F}$ that are deleted in going from $G_{F}$ to $G^{*}$.

Lemma $6 \mathbf{W h p} H_{1}$ has at most $5 \Delta^{2}=o(n)$ edges and has maximum degree at most 19. 
Proof The fact that $H_{1}$ has at most $5 \Delta^{2}$ edges whp follows immediately from Lemma 4 and from the fact that each switching deletes at most 4 edges. Now every edge of $F$ at distance $\geq 2$ from the loop or double edge can be used as one of the two edges destroyed by the two types of switching. Thus vertex $i$ has probability

$$
O\left(\frac{d_{i}}{M_{1}-\Delta^{2}}\right)=O\left(\frac{\Delta}{M_{1}}\right)
$$

of being on an $H_{1}$-edge created by any switching, regardless of the history of the switchings to this point. So if $H_{1}^{\prime}$ is the subgraph of $H_{1}$ induced by these edges (i.e. the non-loops and multiple edges) then for some constant $c>0$, assuming due to Lemma 4 that $G^{*}$ satisfies (18):

$$
\begin{aligned}
\operatorname{Pr}\left(\Delta\left(H_{1}^{\prime}\right) \geq 10\right) \leq n\left(\begin{array}{c}
\Delta^{2}+2 \Delta+2 \log n \\
10
\end{array}\right)\left(\frac{c \Delta}{M_{1}}\right)^{10} \leq \frac{c^{10} \Delta^{30}(\log n)^{10} n}{M_{1}^{10}} \\
\leq \frac{c^{10}(\log n)^{10} M_{2}^{6} n}{\omega^{6} M_{1}^{10}} \leq \frac{c^{10}(\log n)^{10} \Delta^{6} n}{\omega^{6} M_{1}^{4}}=o(1) .
\end{aligned}
$$

after using $\Delta=o\left(n^{1 / 3}\right)$, from (2), and $M_{1} \geq n$.

We can estimate the expected number of vertices incident with $\geq 10$ multiple edges by

$$
n \Delta^{20}\left(\frac{\Delta}{M_{1}}\right)^{10} \leq \frac{n M_{2}^{6}}{\omega^{6} M_{1}^{10}} \leq \frac{n \Delta^{4}}{\omega^{6} M_{1}^{4}}=o(1) .
$$

There are no double loops whp and so whp $\Delta\left(H_{1}\right) \leq 9+9+1=19$.

Remark 3 It is probably a good time to remark that (1) with $k=1$ implies that $\Delta \leq$ $K d n^{1-\alpha}$. And then we get from A2 of Theorem 2 that $\Delta^{2}=o\left(s_{0}\right)$.

Next let $H_{2}$ denote the graph induced by the set of edges added in going from $G_{F}$ to $G^{*}$.

Lemma 7 Whp $H_{2}$ has maximum degree at most 25.

Proof Except for loops and multiple edges, each edge of $H_{2}$ can be paired with an edge of $H_{1}$. Thus $\Delta\left(H_{2}\right) \leq \Delta\left(H_{1}\right)+\Delta_{l}+\Delta_{m}$ where whp $\Delta_{l}=1$ is the maximum number of loops at a vertex and $\Delta_{m}$ is the maximum number of multiple edges at a vertex. Our result follows from $\Delta_{m} \leq 5 \mathrm{whp}$. Indeed,

$$
\operatorname{Pr}\left(\Delta_{m} \geq 6\right) \leq n \Delta^{12}\left(\frac{\Delta}{d n}\right)^{6}=n \frac{\Delta^{18}}{(d n)^{6}} \leq n \frac{M_{2}^{18 / 5}}{(d n)^{6}} \leq n \frac{\Delta^{18 / 5}}{(d n)^{12 / 5}} \leq n^{-1 / 5} .
$$


Let us now see how this affects the argument used in Sections 4.1 and 4.2. Assume now that the edges of $H_{1}$ are deleted and the edges of $H_{2}$ are added.

Going back to Remarks 1 and 3 we see that the argument in Case 1 can handle the deletion of the edges of $H_{1}$ and the addition of the edges in $H_{2}$.

Going back to Remarks 2 and 3 we see that the argument in Case 2 can handle the deletion of the edges of $H_{1}$.

Going back to (17) we see that we can afford to give up $o(n)$ successes in the first $t_{1}$ trials due to deletion of the edges of $H_{1}$.

Going back to (15) we see that we can afford to delete $o(n)$ edges without disconnecting $G_{R B}$.

This completes the proof of Theorem 2.

\section{References}

[1] W. Aiello, F. Chung and L. Lu. A random graph model for power law graphs, Experimental Mathematics 10, (2001), 53-66.

[2] E.A. Bender and E.R. Canfield, The asymptotic number of labelled graphs with given degree sequences, Journal of Combinatorial Theory, Series A 24 (1978) 296-307.

[3] B. Bollobás, A probabilistic proof of an asymptotic formula for the number of labelled regular graphs, European Journal on Combinatorics 1 (1980) 311-316.

[4] B. Bollobás, Random graphs, 2nd Ed., Cambridge Univ. Press, Cambridge, 2001.

[5] C. Cooper, The size of the cores of a random graph with a given degree sequence, Random Structures and Algorithms, 25: 353-375 (2004).

[6] C. Cooper and A.M. Frieze, The size of the largest strongly connected component of a random digraph with a given degree sequence, Combinatorics, Probability and Computing 13 (2004) 319-338.

[7] A.M. Frieze, M. Krivelevich and C. Smyth, On the chromatic number of random graphs with a fixed degree sequence, Combinatorics, Probability and Computing 16 (2007) 733746.

[8] A.M. Frieze and B.G.Pittel, Perfect matchings in random graphs with prescribed minimal degree, Trends in Mathematics, Birkhauser Verlag, Basel (2004) 95-132.

[9] B.D. McKay and N.C. Wormald, Asymptotic enumeration by degree sequence of graphs with degree o( $\left.n^{1 / 2}\right)$, Combinatorica 11 (1991) 369-382. 
[10] B.D.McKay and N.C.Wormald, Asymptotic enumeration by degree sequence of graphs of high degree, European Journal of Combinatorics 11 (1990) 565-580.

[11] B.D. McKay and N.C. Wormald, Uniform generation of random regular graphs of moderate degree, Journal of Algorithms 11 (1990) 52-67.

[12] M. Molloy and B.A. Reed, A Critical Point for Random Graphs with a Given Degree Sequence, Random Structures and Algorithms 6 (1995) 161-180.

[13] M. Molloy and B.A. Reed, The Size of the Largest Component of a Random Graph on a Fixed Degree Sequence, Combinatorics, Probability and Computing 7 (1998) 295-306.

[14] L. Pósa, Hamiltonian circuits in random graphs, Discrete Mathematics 14 (1976) 359364.

[15] N.C. Wormald Models of random regular graphs, Surveys in Combinatorics, London Mathematical Society Lecture Note Series 267, Cambridge University Press, Cambridge, 1999 (J.D.Lamb and D.A.Preece, Eds.), Proceedings of the 1999 British Combinatorial Conference, Cambridge University Press, 239-298. 